\title{
COMENTARIO
}

\section{Índice $h$ (2000-2004) de los científicos ambientales más citados que residen en Chile}

\author{
$h$-index (2000-2004) of the most cited environmental researchers based \\ at Chilean institutions
}

JAIME R. RAU

\begin{abstract}
Laboratorio de Ecología, Departamento de Ciencias Básicas \& Programa IBAM, Universidad de Los Lagos, Casilla 933, Osorno, Chile e-mail: jrau@ulagos.cl
\end{abstract}

Un proyecto de investigación científica podría: (a) generar o no manuscritos, ms; (b) los ms podrían o no ser aceptados para publicación en una revista; (c) la revista podría o no ser de corriente principal, ISI; (d) el factor de impacto de la revista podría o no ser $>1$, umbral usado usualmente para bonificar a autores de universidades chilenas la publicación de sus artículos ISI; (e) el artículo podría o no ser citado y, finalmente, (f) podría solo recibir 1,0 pocas citas, o bien llegar a ser altamente citado (http://isihiglycited.com) y lograr renombre si tiene > 500 citas en acuerdo con la base de datos SPIRES para la física de partículas (http:/ /www.slac.stanford.edu/spires). El impacto de un autor se estima consultando en el "Thompson Scientific-Web of Science (WOS)" las citas primarias que reciben sus artículos registrados en esa base de datos (http:// www.isiknowledge.com).

Recientemente, el físico argentino de la Universidad de California (Campus San Diego) Jorge E. Hirsch (Hirsch 2005) publicó su índice $h$ (accesible también en línea en http:// arxiv.org/abs/physics/0508025), el cual hace equivalentes pocos artículos publicados pero con muchas citas recibidas o muchos artículos pero con pocas citas. Este índice ha sido recibido con entusiasmo (e.g., Ball 2005, Bornmann \& Daniel 2005, Braun et al. 2005, Chapron \& Husté 2006, Cronin \& Meho 2006) y ya se ha escrito una revisión sobre él (Bornmann \& Daniel 2007, agradezco a Lutz Bornmann por enviarme su manuscrito). Un científico tiene un índice $h$ si sus $N_{p}$ artículos tienen al menos $h$ citas cada uno y los otros artículos restantes $\left(N_{p}-h\right)$ no tienen más que $h$ citas cada uno en un período de tiempo $n$ (Hirsch 2005). El índice $h$ será siempre una cantidad menor que el número total de artículos en que se basa (Braun et al. 2005), podrá aumentar linealmente con el tiempo pero no disminuir y será igual a 0 cuando los artículos no acumulen citas (Bornmann \& Daniel 2007).

Hace poco, se han entregado en un capítulo de libro editado por la Academia Chilena de Ciencias (http://www.academia-ciencias.cl) los nombres y el impacto de los 10 autores con residencia en Chile que en Ecología y Ciencias Ambientales han recibido el mayor número de citas para sus artículos ISI publicados durante el quinquenio 2000-2004 (Kalin-Arroyo 2005). A partir de esa base de datos presento aquí el índice $h$ de ocho de ellos (uno figura con dos trabajos y otra no reside en Chile) y lo comparo con el calculado para el año 2005 del cuerpo de editores (rango de $n=9-33)^{1}$ de siete revistas ecológicas de alto impacto (Kelly \& Jennions 2006, agradezco a Clint D. Kelly por hacerme llegar sus datos crudos). El algoritmo a seguir usándose el comando "Times Cited" del WOS (http://isiknowledge.com) para calcular el índice $h$ tiene solo dos etapas: (1) se ordenan por rango las citas que reciben los artículos de un autor y (2) se contabiliza desde el artículo

1 Para efectos comparativos nótese que actualmente la Revista Chilena de Historia Natural tiene $\mathrm{n}=32$ editores, cifra que se encuentra cercana al valor máximo del rango del tamaño de muestra que esos autores utilizaron. 
más citado al menos citado hasta que el recuento no sea mayor que el número total de citas del siguiente artículo.

Los ocho científicos ambientales chilenos aquí considerados publicaron sus 128 artículos durante el período 2000-2004 en 58 revistas ISI (17 de ellos en la Revista Chilena de Historia Natural, ocho en Austral Ecology y solo un artículo para cada una de 36 revistas distintas). Esos artículos recibieron un total de 1.707 citas (ca. 14 citas promedio per cápita) y ambas variables se relacionaron (coeficiente de correlación de rangos de Spearman, $\mathrm{r}_{\mathrm{s}}=0,766$; $\mathrm{P}=0,043 ; \mathrm{n}=8)$. Su número máximo de citas varió entre 20 y 413 por autor. Los factores de impacto (2002-2005) de esas revistas variaron entre 2,212- 29,232 y también se relacionaron con el número máximo de citas recibidas $\left(\mathrm{r}_{\mathrm{s}}=\right.$ $0,838 ; \mathrm{P}=0,027 ; \mathrm{n}=8)$. Su índice $h$ varió entre tres para el valor más bajo y 10 para el valor más alto. En comparación, los valores para los editores de revistas ecológicas de alto impacto variaron entre 9-33 y el de una muestra de 18 de los ecólogos más citados del mundo fue igual a 45 (Kelly \& Jennions 2006). Por lo tanto, los índices $h$ de los científicos ambientales chilenos están más cercanos al valor mínimo del rango de los valores del índice $h$ calculado por Kelly \& Jennions (2006) para los editores de las revistas ecológicas de mayor impacto a nivel mundial.

Se ha sugerido que en la medida que más se publica así también disminuye la probabilidad de recibir citas por artículo (Hirsch 2005, Leimu \& Koricheva 2005). La asociación entre el número de artículos ISI publicados y el número de aquellos que no fueron citados en el período de tiempo aquí establecido fue positiva y estadísticamente significativa $\left(\mathrm{r}_{\mathrm{s}}=0,876 ; \mathrm{P}=\right.$ $0,020 ; \mathrm{n}=8)$. La asociación con el índice $h$ también lo fue $\left(\mathrm{r}_{\mathrm{s}}=0,800 ; \mathrm{P}=0,034 ; \mathrm{n}=8\right)$. De la información original presentada por Kalin-Arroyo (2005) se aprecia que solamente la mitad de los autores más productivos en Ciencias Ambientales resultaron ser también los más citados. En el caso de la Ecología evidencias recientes sugieren que tanto el índice $h$ (Kelly \& Jennions 2006) como la frecuencia de citas (Leimu \& Koricheva 2005) no se asocian primariamente con las características de un estudio per se sino que se asocian más a factores sociales, tales como las características de los autores, el prestigio de las revistas y el valor retórico de las hipótesis probadas.

Comparado a otros indicadores bibliométricos (productividad dada por el número de artículos publicados, visibilidad o impacto dado por el número de citas recibidas), el índice $h$ consideró en un solo valor ambos factores puesto que se relacionó tanto con el número total de citas $\left(\mathrm{r}_{\mathrm{s}}=0,747 ; \mathrm{P}=0,048 ; \mathrm{n}=\right.$ 8) y más aún con el número total de artículos $\left(\mathrm{r}_{\mathrm{s}}=0,939 ; \mathrm{P}=0,013 ; \mathrm{n}=8\right)$. Una de las potenciales limitantes de la aplicación de este índice sería la dificultad en comparar investigadores al inicio y final de su carrera académica (Hirsch 2005). Para analizar este efecto utilicé las bases de datos de la Comisión Nacional de Investigación Científica y Tecnológica (http://www.conicyt.cl/bases/ fondecyt/index.html) y de la Academia Chilena de Ciencias (http://www.academia-ciencias.cl). De ellas obtuve el año en que los ocho científicos ambientales más citados con residencia en Chile obtuvieron sus respectivos doctorados. Asumiéndose que la publicación de los primeros artículos se produce en esta etapa de su carrera profesional (Hirsch 2005) relacioné estos años (variaron entre 1966 y 2002) y el índice de Hirsch no encontrando ninguna asociación $\left(\mathrm{r}_{\mathrm{s}}=0,267 ; \mathrm{P}=0,480 ; \mathrm{n}=\right.$ 8). Por lo tanto, concluyo que al menos para esta muestra el factor "edad académica" no influyó sobre el valor del índice $h$. Basado en mis análisis estimo que este índice puede ser de utilidad comparativa para evaluar la productividad científica de los científicos ambientales en Chile y otras regiones del mundo.

\section{AGRADECIMIENTOS}

Agradezco a la Dirección de Investigación de la Universidad de Los Lagos por financiar los costos de publicación de este trabajo.

\section{LITERATURA CITADA}

BALL P (2005) Index aims for fair ranking of scientists Nature 436: 900.

BORNMANN L \& H-D DANIEL (2005) Does the h-index for ranking of scientists really work? Scientometrics 65: 391-392.

BORNMANN L \& H-D DANIEL (2007) What do we know about the $h$ index? Journal of the American 
Society for Information Science and Technology 58: 1381-1385.

BRAUN T, W GLÄNZEL \& A SCHUBERT (2005) A Hirsch-type index for journals. The Scientist 19: 8 .

CRONIN B \& L MEHO (2006) Using the $h$-index to rank influential Information scientists. Journal of the American Society for Information Science and Technology 57: 1275-1278.

CHAPRON G \& A HUSTÉ (2006) Open, fair, and free journal ranking for researchers. Bioscience 56: 558559.

HIRSCH JE (2005) An index to quantify an individual's scientific research output. Proceedings of the National Academy of Sciences 102: 16569-16572.
KALIN-ARROYO MT (2005) Ecología y ciencias ambientales. En: Ureta T, J Babul, S Martínez \& J Allende (eds) Análisis y proyecciones de la ciencia chilena-2005: 295-331. Academia Chilena de Ciencias. Santiago, Chile. http://www.academiaciencias.cl/docs/noticias/estudio.pdf

KELLY CD \& MD JENNIONS (2006) The $h$ index and career assessment by numbers. Trends in Ecology and Evolution 21: 167-170.

LEIMU R \& KORICHEVA (2004) What determines the citation frequency of ecological papers? Trends in Ecology and Evolution 20: 28-32. 
